\title{
Stop Crying over Spilled Milk, Thanks to Recent Reports Which Suggest Novel Therapeutic Options for Existing Aldose reductase Inhibitors
}

\section{Kota V Ramana*}

Department of Biochemistry and Molecular Biology, University of Texas Medical Branch, Galveston, USA

Aldose reductase is an enzyme that catalyzes the first and rate limiting step of polyol pathway of glucose metabolism. It uses $\mathrm{NAPDH}$ as a co-factor to reduce various physiologically important polyols to corresponding alcohols. During hyperglycemia a major portion $(>30 \%)$ of glucose metabolism is carried out by this pathway leading to the production of sorbitol and fructose [1]. Sorbitol being impermeable to the biological membranes accumulates inside and cause osmotic stress which is considered as one of the major causes of diabetic complications. Because of this property, AR was identified as the chief progenitor of hyperglycemic injury during diabetes [2]. Subsequently, several AR inhibitors (ARIs) have been synthesized and tested for their efficacy in several secondary diabetic complications such as cataractogenesis, retinopathy, neuropathy and nephropathy. ARIs that belongs to spirohydantoins (such as sorbinil, minalrestat, ranirestat and fidarestat) and carboxylic acid derivatives (such as tolrestat, zopolrestat, zenarestat, ponalrestat, and epalrestat), and various natural flavonoids (such as quercetin and resveratrol) have been assessed for their efficacy in the treatment of diabetic complications $[3,4]$. Buoyed by the experimental animal data documenting protection from key features of diabetic tissue injury and the generation of continued support for a critical involvement of AR in diabetic complications, several ARIs are gone up to phase-III clinical trials in the United States and other places. Although these inhibitors have been found to be safe for human use in the clinical trials, they have not been approved for use in the United States may be due to lack of substantive clinical improvement and moderate side effects including skin rashes and liver toxicity. Exception to this, ARIs such as fidarestat was found to be highly specific and found to be relatively safe for human use in a 52 week long clinical study for diabetic neuropathy [5]. Eparelstat is only the AR inhibitor that is in the market in Japan for the treatment of diabetic complications such as nephropathy [6]. Now the interesting question is why ARIs failed at the clinical studies? The ARIs were tested based on their ability to prevent the formation of sorbitol. Therefore, the polyol hypothesis provided a simple testable paradigm of hyperglycemic complications, though several key observations during diabetes were not explained and explored. For instance, in several tissues the intracellular accumulation of sorbitol is not high enough to cause significant osmotic stress even after prolonged hyperglycemia. Furthermore, the high efficacy of antioxidants in preventing cataractogenesis, without preventing sorbitol accumulation, suggested that oxidative stress may be an important feature of hyperglycemic injury [7]. In addition to polyol accumulation, other metabolic changes have also been suggested to account for hyperglycemic injury. Of these, non enzymatic glycosylation leading to the accumulation of advanced glycosylation products (AGEs) and alterations in protein kinase $\mathrm{C}$ (PKC) and myo-inositol levels have received the most attention. Nonetheless, the mechanistic reasons why ARIs failed to prevent diabetic complications in clinical studies continue to be a matter of debate. This issue is of more than mere academic interest, since in the absence of a detailed understanding the physiological functions of AR in normal and hyperglycemic conditions, the use of ARIs could not be advocated with confidence for diabetic complications.
Based on the recent investigations from our lab, as well as reports from other investigators, it is now clear that the physiological role of AR is not simply reducing polyols but it exhibits wide spread substrate specificity $[8,9]$. The isolated, homogenous enzyme displayed poor affinity for glucose, with a $\mathrm{Km}$ between 50 to $100 \mathrm{mM}$ and the kinetic properties of AR were unlike those of other glucose-metabolizing enzymes. These anomalies were brought into sharp focus when the $\mathrm{X}$ ray crystal structure of the AR showed high hydrophobicity at the putative substrate binding domain which essentially precludes efficient carbohydrate reduction, and suggests that hydrophobic aldehydes are likely to be the preferred substrates [1]. The most obvious endogenous source of hydrophobic aldehydes is oxidative stress-induced lipid peroxidation, a generally observed process in number of pathological disease conditions. Large amounts of toxic aldehydes are formed during free radical-mediated peroxidation of lipids. Several of these aldehydes display high toxicity, conjugate with cellular glutathione and other important proteins and could mediate some of the biological effects ascribed to their radical precursors. The observation that AR may represent an important metabolic route for the reduction of lipid peroxidation derived aldehydes (such as 4-hydroxy-trans-2 nonenal; 4-HNE) and their glutathione conjugates (such as GS-HNE) with Km in low 10-30 $\mu \mathrm{M}$ range provided an important breakthrough in our understanding of the physiological role of this enzyme $[10,11]$. In addition, AR has been shown to reduce a number of other non polyol substrates such as steroids, corticosteroids, unsaturated aldehydes, aromatic aldehydes, phospholipids, and base propanals [1]. Most importantly, lipid aldehydes are key participants in cellular signaling events that cause cell growth, differentiation and death ultimately leading to tissue damage and dysfunction in various life threatening diseases. AR that catalyzes these aldehydes could play a major role in regulating the cellular signals and pathologies associated with those. Indeed, recent cell culture and animals studies have identified that AR mediates signals initiated by various cytokines, growth factors, chemokines, bacterial endotoxin and allergens $[12,13]$. Specifically, inhibitors of AR have shown to inhibit activation of redox sensitive transcription factors such as NF-kB, AP1 which are known to transcribe various inflammatory markers involved in various inflammatory complications. These results suggest that AR inhibitors that regulate activation of transcription factors could alter pathologies where increased expressions of these transcription factors are major

*Corresponding author: Kota $\mathrm{V}$ Ramana, Department of Biochemistry and Molecular Biology, University of Texas Medical Branch, Galveston, USA, E-mail: kvramana@utmb.edu

Received August 16, 2012; Accepted August 18, 2012; Published August 24 2012

Citation: Ramana KV (2012) Stop Crying over Spilled Milk, Thanks to Recent Reports Which Suggest Novel Therapeutic Options for Existing Aldose reductase Inhibitors. J Biomol Res Ther 1:e105. doi:10.4172/2167-7956.1000e105

Copyright: (c) 2012 Ramana KV. This is an open-access article distributed under the terms of the Creative Commons Attribution License, which permits unrestricted use, distribution, and reproduction in any medium, provided the original author and source are credited. 
culprits. Without a doubt, recent studies indicate that inhibitors of AR prevent various inflammatory diseases such as colon cancer, sepsis, asthma, cardiomyopathy, atherosclerosis and uveitis in experimental animal models. These studies backed up by strong evidence obtained using tissue culture system using human as well as rodent cells suggest that AR plays a pivotal role in the patho-physiology of inflammatory diseases including cancer. AR has been shown to be over-expressed in a number of human cancers including colon, lung, pancreas and breast cancers [14]. The studies that show AR inhibition prevent colon cancer growth and metastasis in nude mouse models suggest that ARIs could be used as chemo-preventive drugs [15]. Further, ARIs have shown to prevent angiogenesis, a process required for tumor growth and metastasis, by using cell culture system and rat matrigel plug model. These studies indicate that AR inhibition could be anti-angiogenic [15]. In addition, the anti-inflammatory role of AR inhibitors has been shown in different indications. For example, ARIs such as sorbinil and fidarestat prevent bacterial endotoxin-induced inflammatory response, cardiac dysfunction, lung toxicity in mice [16,17]. Similarly, AR inhibition prevents inflammatory cytokine production in cecum ligation puncture model of sepsis in mice [16]. Most importantly, AR inhibitor decreases the LPS-induced mortality in mice. These results suggest that ARIs could be developed to prevent sepsis complications due to bacterial infections. Recently, ARIs have been shown to prevent ocular inflammatory complications such as uveitis. Antiocular inflammatory actions of AR inhibition have been shown in endotoxin as well as autoimmune-induced uveitis models in rodents [18]. Prevention of atherosclerosis and restenosis in animal models suggest that ARIs possess anti-proliferative effects [19]. Recent studies also demonstrate AR inhibitors as anti-asthmatic drugs. ARIs such as sorbinil, fidarestat and zopolrestat have been shown to prevent allergic airway inflammation in mouse models of ragweed pollen extract and ovalbumin-induced asthma complications [20)] Most of the studies performed using ARIs have been confirmed by studies using transgenic or knockout mouse models. These studies thus strongly indicate the potential of ARIs in various inflammatory complications.

Inflammatory disorders such as asthma, COPD, cancer (colon, breast, prostrate, lung), metastasis of cancer and sepsis are the major causes of morbidity and mortality worldwide. Currently, there is no drug that can adequately prevent and/or treat these disorders. During last decade various approaches have been considered for the development of ARIs in preventing such life threatening disorders. These efforts made a remarkable impact on the research realm for identifying novel therapeutics for existing drugs. The new therapeutic indications established by ARIs suggest the necessity of developing these inhibitors to treat other inflammatory complications than diabetes. It is not appropriate to desert ARIs as they did not show promising results in diabetic neuropathy or retinopathy in the clinical trials. Instead of thinking of them as spilled milk, recent studies provided a wonderful opportunity to explore more about ARIs in different directions. With few additional proof-of-concept studies using human samples and preclinical studies to demonstrate best route of application, quality and efficacy, one can see the brighter side of darkness in this field. From the standpoint of view, the current state of research and development of ARIs for novel therapeutic uses demonstrate a symbiosis of the recent achievements and previous controversies, a typical route of our progress in the new drug development.

\section{References}

1. Srivastava SK, Ramana KV, Bhatnagar A (2005) Role of aldose reductase and oxidative damage in diabetes and the consequent potential for therapeutic options. Endocr Rev 26: 380-392.
2. Kinoshita JH, Nishimura C (1988) The involvement of aldose reductase in diabetic complications. Diabetes Metab Rev 4: 323-337.

3. Tomlinson DR, Willars GB, Carrington AL (1992) Aldose reductase inhibitors and diabetic complications. Pharmacol Ther 54: 151-194.

4. Del Corso A, Cappiello M, Mura U (2008) From a dull enzyme to something else: facts and perspectives regarding aldose reductase. Curr Med Chem 15: 1452-1461.

5. Giannoukakis N (2003) Fidarestat. Sanwa Kagaku/NC Curex/Sankyo. Curr Opin Investig Drugs 4: 1233-1239.

6. Ramirez MA, Borja NL (2008) Epalrestat: an aldose reductase inhibitor for the treatment of diabetic neuropathy. Pharmacotherapy 28: 646-655.

7. Srivastava SK, Ansari NH (1988) Prevention of sugar-induced cataractogenesis in rats by butylated hydroxytoluene. Diabetes 37: 1505-1508.

8. Ramana KV (2011) ALDOSE REDUCTASE: New Insights for Old Enzyme. Biomol Concepts 2: 103-114.

9. Alexiou P, Pegklidou K, Chatzopoulou M, Nicolaou I, Demopoulos VJ (2009) Aldose reductase enzyme and its implication to major health problems of the 21 (st) century. Curr Med Chem 16: 734-752.

10. Dixit BL, Balendiran GK, Watowich SJ, Srivastava S, Ramana KV, et al. (2000) Kinetic and structural characterization of the glutathione-binding site of aldose reductase. J Biol Chem 275: 21587-21595.

11. Ramana KV, Dixit BL, Srivastava S, Balendiran GK, Srivastava SK, et al. (2000) Selective recognition of glutathiolated aldehydes by aldose reductase. Biochemistry 39: 12172-12180.

12. Srivastava SK, Yadav UC, Reddy AB, Saxena A, Tammali R, et al. (2011) Aldose reductase inhibition suppresses oxidative stress-induced inflammatory disorders. Chem Biol Interact 191: 330-338.

13. Ramana KV, Srivastava SK (2010) Aldose reductase: a novel therapeutic target for inflammatory pathologies. Int J Biochem Cell Biol 42: $17-20$.

14. Saraswat M, Mrudula T, Kumar PU, Suneetha A, Rao Rao TS, et al. (2006) Overexpression of aldose reductase in human cancer tissues. Med Sci Monit 12: CR525-529.

15. Tammali R, Srivastava SK, Ramana KV (2011) Targeting aldose reductase for the treatment of cancer. Curr Cancer Drug Targets 11: 560-571.

16. Pandey S, Srivastava SK, Ramana KV (2012) A potential therapeutic role for aldose reductase inhibitors in the treatment of endotoxin-related inflammatory diseases. Expert Opin Investig Drugs 21: 329-339.

17. Takahashi K, Mizukami H, Kamata K, Inaba W, Kato N, et al. (2012) Amelioration of acute kidney injury in lipopolysaccharide-induced systemic inflammatory response syndrome by an aldose reductase inhibitor fidarestat. PLoS One 7: e30134.

18. Yadav UC, Srivastava SK, Ramana KV (2010) Understanding the role of aldose reductase in ocular inflammation. Curr Mol Med 10: 540-549.

19. Ramasamy R, Goldberg IJ (2010) Aldose reductase and cardiovascular diseases, creating human-like diabetic complications in an experimental model. Circ Res 106: 1449-1458.

20. Ramana KV, Yadav UC, Calhoun WJ, Srivastava SK (2011) Current prospective of aldose reductase inhibition in the therapy of allergic airway inflammation in asthma. Curr Mol Med 11. 599-60 